\section{THU0033 ALTERATIONS IN THE PHENOTYPIC LANDSCAPE AND SPECIFICITY OF CD4+ T CELLS IN CCP+ AT RISK SUBJECTS BEFORE THE ONSET OF RHEUMATOID ARTHRITIS}

C. Rims ${ }^{1}$, V. Muir ${ }^{2}$, K. Deane ${ }^{3}$, S. Nagpal ${ }^{4}$, N. Rao ${ }^{4}$, F. Baribaud ${ }^{4}$, G. Vratsanos ${ }^{4}$, V. M. Holers ${ }^{3}$, P. Linsley ${ }^{2}$, E. A. James ${ }^{1}$, J. Buckner ${ }^{1}{ }^{1}{ }^{1}$ Benaroya Research Institute, Translational Research, Seattle, United States of America; ${ }^{2}$ Benaroya Research Institute, Bioinformatics, Seattle, United States of America; ${ }^{3}$ University of Colorado School of Medicine, Division of Rheumatology, Aurora, United States of America; ${ }^{4}$ Janssen Pharmaceuticals, Spring House, United States of America

Background: The "Targeting Immune Responses for Prevention of RA" (TIP-RA) collaboration studies individuals at high risk for developing rheumatoid arthritis (RA) because of serum anti-citrullinated protein antibody (ACPA) positivity in absence of arthritis at baseline, and is focused on defining how they transition from at-risk to classifiable disease. One potential mechanism is the expansion of antigen specific $T$ cells that recognize self-antigens and acquisition of disease associated T cell phenotypes. ACPA emerge years prior to clinically apparent disease and subsequently increase in their titer and breadth of specificity. However, few studies have characterized $T$ cells during this transition.

Objectives: To identify features associated with progression to RA by examining the specificity and surface phenotype of CD4+T cells in individuals from the TIP-RA cohort by HLA class II tetramer staining and multi-parameter flow cytometry.

Methods: Tetramer staining and flow cytometry were performed on peripheral blood samples from a baseline visit from CCP3- controls $(n=34), C C P 3+$ at-risk $(n=26), C C P 3+$ positive individuals who transitioned in the near-term to RA (called "RA converters", $n=4$ ), and seropositive early-RA ( $n=21)$. Our staining panel allowed us to measure the frequencies of $T$ cells specific for citrullinated alpha-enolase, aggrecan, cartilage intermediate layer protein (CILP), fibrinogen and vimentin. We then applied both supervised phenotyping and a cluster-based computational approach to compare the phenotypic landscape and specificity of antigen specific and total CD4+ T cells in each cohort.

Results: We observed higher overall frequencies of $T$ cells that recognize citrullinated epitopes in CCP3+ at-risk subjects than CCP- controls $(p<0.05)$. Among the individual specificities, elevated frequencies prior to disease onset were most prominent for CILP specific T cells. Supervised phenotypic analysis revealed an increase in CCR4+ CD4+ T cells in CCP3+ at risk subjects $(p<0.001)$ and a corresponding decrease in CXCR3+ CD4+ T cells that was most pronounced in RA converters and seropositive early-RA $(p<0.05)$. Cluster-based phenotypic analysis defined ten distinct phenotypic states present within all subjects. Each of these ten immunotypes contained $T$ cells that recognize citrullinated epitopes. However, the predominan immunotype varied for different antigens. During progression, the frequencies of Ag specific $\mathrm{T}$ cells diminished when onset was imminent, but rebounded shortly after diagnosis. Concomitantly, Ag specific $\mathrm{T}$ cells with memory phenotypes were diminished, but subsequently reverted to TSCM, Th1, and Th1-17 like phenotypes. Conclusion: Our data show that disease associated changes in the antigen specificity of CD4+ T cells are present in CCP3+ at-risk subjects. Furthermore, the number of antigen specific $T$ cells and their phenotype are perturbed before the onset of symptoms and development of classified RA. These findings support a continuum of immunologic changes that underlie risk and drive disease, motivating new approaches for early intervention.

Acknowledgments: We gratefully acknowledge the Targeting Immune Responses for Prevention of Rheumatoid Arthritis (TIP-RA) for designing and executing this collaborative study

Disclosure of Interests: Cliff Rims: None declared, Virginia Muir: None declared, Kevin Deane Grant/research support from: Janssen, Consultant of: Inova, ThermoFisher, Janseen, BMS and Microdrop, Sunil Nagpal Shareholder of: Janssen Pharmaceuticals, Employee of: Janssen Pharmaceuticals, Navin Rao Shareholde of: Janssen Pharmaceuticals, Employee of: Janssen Pharmaceuticals, Frederic Baribaud Shareholder of: Janssen Research \& Development, LLC, Employee of: Janssen Research \& Development, LLC, George Vratsanos Shareholder of: Janssen Pharmaceuticals, Employee of: Janssen Pharmaceuticals, V. Michae Holers Grant/research support from: Janssen, Celgene, and BMS, Peter Linsley Consultant of: BMS, Eddie A. James Grant/research support from: Janssen, Pfizer, Sanofi, Novartis, Jane Buckner Grant/research support from: Bristol-Myers Squibb, Janssen

DOI: 10.1136/annrheumdis-2020-eular.1918

\begin{tabular}{|l|l}
\hline THU0034 & AUTOREACTIVE B CELLS ESCAPE PERIPHERAL \\
CHECKPOINT IN ANCA-ASSOCIATED VASCULITIS \\
AND SJÖGREN'S SYNDROME
\end{tabular}

N. Chriti ${ }^{1}$, M. Boudigou' ${ }^{1}$, E. Porchet ${ }^{1}$, J. O. Pers ${ }^{1}$, S. Hillion ${ }^{1}$, D. Cornec ${ }^{1}$ ${ }^{1}$ UMR1227, Lymphocytes B et Autoimmunité, Université de Brest, Inserm, Brest, France, BREST, France
Background: B cells play a central role in many autoimmune diseases (AIDs) including ANCA-associated vasculitis (AAV) and primary Sjögren's syndrome (pSS). Most of the research that has been conducted on AID has focused on the production, secretion, and pathogenicity of auto-antibodies, but little is known on the characteristics of autoreactive $B$ cells in humans.

Objectives: This study aims at characterizing circulating autoantigen (PR3 ou SSA)-specific B-cells in patients with AAV and pSS compared to healthy subjects to better understand their role in the natural and pathological autoimmunity and define the mechanisms leading to the breakdown of self-tolerance in patients with AID.

Methods: First, we developed a new flow-cytometry method to detect circulating auto-reactive B cell based on the specificity of their B-cell receptor (BCR). To study surface phenotype of specific B cells by flow cytometry, blood samples were collected from patient with PR3-ANCA AAV, pSS and from healthy subjects Functional analysis of antigen-specific $B$ cells was also elicited by in vitro analysis of their capacity to secrete immunoglobulins against SSA or PR3 antigens by ELISPOT

Results: Phenotype analysis showed that antigen-specific B cells in patients have a memory phenotype compared with healthy controls (5 to $9 \%$ are IgG-expressing memory $\mathbf{B}$ cells). It suggests that in AID, theses auto-reactive cells are able to differentiate into IgG isotype-switched cells and escape periphera tolerance checkpoint but not in healthy subjects. Interestingly, Natural auto-reactive $B$ cells are able to secrete only IgM isotype autoantibodies upon in vitro stimulation but not IgG class switched antibodies. In order to better understand what differentiates auto-reactive B cells and the mechanisms leading to pathological autoimmunity, a genomic analysis of the antibody repertoire as well as a transcriptional profiling of these cells by single-cell RNA seq is ongoing to understand further the differences of these autoreactive $B$ cells between healthy subjects and patients with AIDs.

Conclusion: We developed a technology to identify and isolate antigen-specific $B$ cells from the peripheral blood of patients with AID. Our results suggest that autoreactive $B$ cells escape peripheral tolerance checkpoint and are able to differentiate into IgG isotype-switched cells in patients with AIDs but not in healthy subjects.

References:

[1] D. Cornec, A. Berti, A. Hummel, T. Peikert, J.-O. Pers, et U. Specks, « Identification and phenotyping of circulating autoreactive proteinase 3-specific $B$ cells in patients with PR3-ANCA associated vasculitis and healthy controls ", J. Autoimmun., vol. 84, p. 122-131, 2017

[2] P. F. Kerkman et al., " Identification and characterisation of citrullinated antigen-specific $B$ cells in peripheral blood of patients with rheumatoid arthritis ", Ann. Rheum. Dis., vol. 75, no 6, p. 1170-1176, juin 2016, doi: 10.1136/ annrheumdis-2014-207182.

Acknowledgments: with support of vasculitis foundation, CSL Berhing, SFR

Disclosure of Interests: None declared

DOI: 10.1136/annrheumdis-2020-eular.5748

\section{THU0035 OX40L EXPRESSING NEUTROPHILS INDUCE CD4 T FOLLICULAR AND PERIPHERAL HELPER CELL DIFFERENTIATION IN SYSTEMIC LUPUS ERYTHEMATOSUS}

A. Pappalardo ${ }^{1}$, E. Wojciechowski ${ }^{1}$, I. Odriozola ${ }^{2}$, I. Douchet ${ }^{1}$, N. Merillon ${ }^{1}$, A. Boizard-Moracchini ${ }^{1}$, P. Duffau ${ }^{1,3}$, E. Lazaro ${ }^{1,3}$, M. A. Doucey ${ }^{4}$, L. Mbow ${ }^{4}$, C. Richez ${ }^{1,2}$, P. Blanco ${ }^{1,5} .{ }^{1}$ Bordeaux University, CNRS-UMR5164, ImmunoConcEpt, Bordeaux, France; ${ }^{2}$ Bordeaux University Hospital, Rheumatology Department, Bordeaux, France; ${ }^{3}$ Bordeaux University Hospital, Internal Medicine Department, Bordeaux, France; ${ }^{4}$ Ichnos Sciences, Epalinges, Switzerland; ${ }^{5}$ Bordeaux University Hospital, Immunology and Immunogenetic Department, Bordeaux, France

Background: Neutrophils have been described as potent antigen-presenting cells able to activate $\mathrm{T}$ cells by MHC/TCR interaction and costimulatory molecules in tumor immunity. However, little is known about the direct interaction between neutrophils and CD4 T cells with respect to systemic lupus erythematosus (SLE). We have previously shown that OX40L expressed by monocytes from SLE patients promote the differentiation of naïve and memory cells into IL21 secreting $T$ cells that are able to help $B$ cells ${ }^{1,2}$.

Objectives: In this study, we investigate OX40L expression on neutrophils from SLE patients and contribution of these $\mathrm{OX}_{40 \mathrm{~L}}{ }^{+}$neutrophils in SLE pathogenesis to modulation of the $\mathrm{B}$ cell helper role of CD4 T cells.

Methods: Surface expression of co-stimulatory molecules (OX40L, ICOSL, GITRL, 4-1BBL) on neutrophils from SLE patients and healthy donors (HD) was measured by flow cytometry (FC). Neutrophils from HD were stimulated with TLR7 or TLR8 agonists and IFNa after 5 hours of culture, OX40L expression was measured by FC and Western Blotting. CD4 T cells were cultured with the stimulated neutrophils for 3 days. At the end of the co-culture, percentages of 
IL21-expressing T follicular (Tfh) and peripheral helper (Tph) cells measured by FC. These generated T cells were also cultured in the presence of memory $B$ cells. After 5 days of co-culture, plasmablast generation and Ig levels were assessed by FC and ELISA, respectively. Inhibition of OX40-OX40L interaction in vitro was achieved using ISB 830, a novel anti-OX40 mAb currently used in clinical trials.

Results: Among the co-stimulatory molecules tested, percentages of $\mathrm{OX}_{40 \mathrm{~L}^{+} \text {neu- }}$ trophils in SLE $(n=54)$ were increased compared to HD $(n=25)($ mean + SD: HD = $1,34 \% \pm 1.62$ vs SLE $=4,53 \% \pm 8.1 ; p=0.29$ ). OX40L expression positively correlated with SLE disease activity score (SLEDAI) $(p=0,04 ; r=0,31)$ and with anti-DNA antibodies $(p=0,04, r=0,33)$. Of note, the percentage of $\mathrm{OX}_{40 \mathrm{~L}^{+} \text {neutrophils was }}$ higher in anti-sm-RNP ${ }^{+}$patients $(n=16$, mean $=9 \% \pm 9.8)$, compared to anti-smRNP' patients $(n=27$, mean $=1,4 \% \pm 2.5 ; p=0,02)$. The percentage of $\mathrm{OX}_{40 \mathrm{~L}^{+}}$ neutrophils was higher in patients with class III or IV lupus nephritis, and inflammatory infiltrate within the kidney biopsy disclosed $\mathrm{OX}_{40 \mathrm{~L}^{+}}$neutrophils, in close contact with T cells. Neutrophils from HD express OX40L with TLR8 agonist, or IFNa priming followed by TLR7 agonist. When memory CD4 T cells were cultured in the presence of TLR8-stimulated neutrophils, the proportion of IL21-expressing Tfh $\left(\mathrm{CXCR} 5^{+} \mathrm{PD} 1^{+}\right)$and Tph $\left(\mathrm{CXCR} 5^{-} \mathrm{PD} 1^{\mathrm{hi}}\right)$ were increased, compared to culture with unstimulated neutrophils. This process was dependent on OX40-OX40L interactions, since in vitro treatment with the anti-OX40 blocking antibody ISB 830, inhibited the differentiation of memory T cells into Tfh and Tph. Both generated Tfh and Tph were able to promote the differentiation of memory B cells into lg-secreting plasmablasts.

Conclusion: Our results disclose an unprecedented phenomenon where cross-talk between TLR7/8-activated neutrophils and CD4 lymphocytes operates through OX40L-OX40 costimulation, and neutrophils promote the differentiation of pro-inflammatory Tfh and Tph, as well as IL21 production. Therefore, OX40L/OX40 should be considered as a potentially therapeutic axis in SLE patients.

References:

[1] Jacquemin et al. Immunity 2015 ;

[2] Jacquemin et al. JCI Insight 2018

Disclosure of Interests: Angela Pappalardo Grant/research support from: Ichnos Sciences, Elodie Wojciechowski: None declared, Itsaso Odriozola: None declared, Isabelle Douchet: None declared, Nathalie Merillon: None declared, Andrea Boizard-Moracchini: None declared, Pierre Duffau: None declared, Estibaliz Lazaro: None declared, Marie-Agnes Doucey Employee of: Ichnos Sciences, Lamine Mbow Employee of: Ichnos Sciences, Christophe Richez Consultant of: Abbvie, Amgen, Mylan, Pfizer, Sandoz and UCB., Patrick Blanco Grant/research support from: Ichnos Sciences

DOI: 10.1136/annrheumdis-2020-eular.2739

\section{THU0036 \\ FIRST-IN-HUMAN TRIAL OF BCMA-CD19 COMPOUND CAR IN THE TREATMENT OF AUTOANTIBODY MEDIATED DISORDERS}

F. Liu ${ }^{1}$, H. Zhang ${ }^{2}$, X. Wang ${ }^{1}$, J. Feng ${ }^{2}$, Y. $\mathrm{Cao}^{3}$, Y. Su${ }^{1}$, M. Wada ${ }^{3}$, Y. Ma ${ }^{4}$, Y. Ma ${ }^{3} .{ }^{1}$ Chengdu Military General Hospital, Hematology, Chengdu, China; ${ }^{2}$ Peking University Shenzhen Hospital, Shenzhen, China; ${ }^{3}$ iCell Gene Therapeutics LLC, Stony Brook, NY, United States of America; ${ }^{4}$ iCAR Bio Therapeutics Ltd, Zhongshan, China

Background: Donor-specific anti-HLA antibodies (DSAs) are antibodies in the recipient directed against donor class I/II HLA antigens. The existence of DSAs before allogenic hematopoietic stem cell transplantation (AHSCT) are known to cause primary graft failure. Currently there's no established method of DSA desensitization due to the long half-life of plasma cells.

Systemic lupus erythematosus (SLE) is a heterogeneous autoimmune disease involving in multiple organ systems mediated by numerous autoantibodies. Recent results have shown that depletion of B cells by CD19 CAR-T cells effectively reversed some manifestations in two SLE mouse models. However, plasma cells could be spared with single CD19 CAR-T cells, and peripheral circulating anti-DNA IgG and IgM autoantibodies remain elevated or increased in treated mice.

Objectives: We present the efficacy of BCMA-CD19 compound CAR (cCAR), which target on antibody- producing "root", both B cells and plasma cells in preclinical study and in our first-in-human phase 1 clinical trial.

Methods: We constructed a BCMA-CD19 cCAR composed of a complete BCMACAR fused to a complete CD19 CAR, separated by a self-cleaving P2A peptide. We assessed the functional activity of CCAR in co-culture assay with multiple cell lines. We also verified cCAR efficacy with two mouse models, injected with either BCMA-expressing MM.1S cells or CD19-expressing REH cells. In our phase 1 clinical trial, we enrolled patients with hematologic malignancies with antibody mediated disorders.

Results: BCMA-CD19 cCAR exhibited robust cytotoxic activity against the K562 cells engineered to express either CD19 or BCMA in co-culture assays, indicating the ability of each complete CAR domain to specifically lyse target cells. In mouse model study, cCAR-T cells were able to eliminate tumor cells in mice injected with MM.1S cells and REH cells, indicating that both BCMA and CD19 are specifically and equally lysing B cells and plasma cells in vivo, making BCMA-CD19 cCAR a candidate for clinical use.

In our first-in-human clinical trial, the first case is a 48 -year-old female patient having resistant B-ALL with high DSA titers. She exhibited complete remission of B-ALL at day 14 post-CAR T treatment. MFI of DSA dropped from 7800 to 1400 at 8 weeks post cCAR treatment, the reduction percentage was approximately $80 \%$ (Figure 1). The patient had no CRS, and no neurotoxicity was observed.

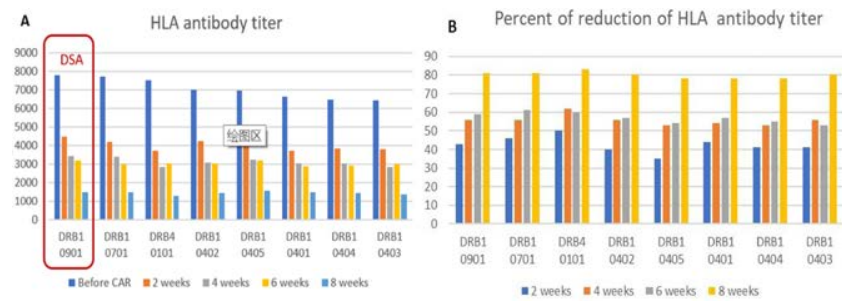

Figure 1. 1. A) MFI of DSA and other HLA antibodies before and at different time points after cCAR T infusion. B) the percent reduction post-transfusion of cCAR T cells at different time points.

The second case is a 41-year-old female patient having a refractory diffuse large $B$ cell lymphoma with bone marrow (BM) involvement. Furthermore, she has a 20 years of SLE, with manifestation of fever dependent of corticosteroids. On day 28 after cCAR treatment, PET/CT scan showed CR, and BM turned negative. In addition, she is independent of steroids, has no fever and other manifestations C3/C4 are within normal ranges, and all the ANA dropped significantly, especially the nuclear type ANA, which turned from $>1: 1000$ to be negative at day 64 . She had Grade 1 CRS but with no neurotoxicity observed. The absence of B cells and plasma cells persisted more than 5 months post CAR therapy.

Conclusion: Our first in human clinical trial on BCMA-CD19 cCAR demonstrated profound efficacy in reducing DSA levels in an AHSCT candidate and ANA titer in a SLE patient. There was strong clinical evidence of depletion of antibody-producing roots, B-cells and plasma cells in both patients. Our results further suggested that BCMA-CD19 CCAR has the potential to benefit patients receiving solid organ transplants or those with other antibody-mediated diseases.

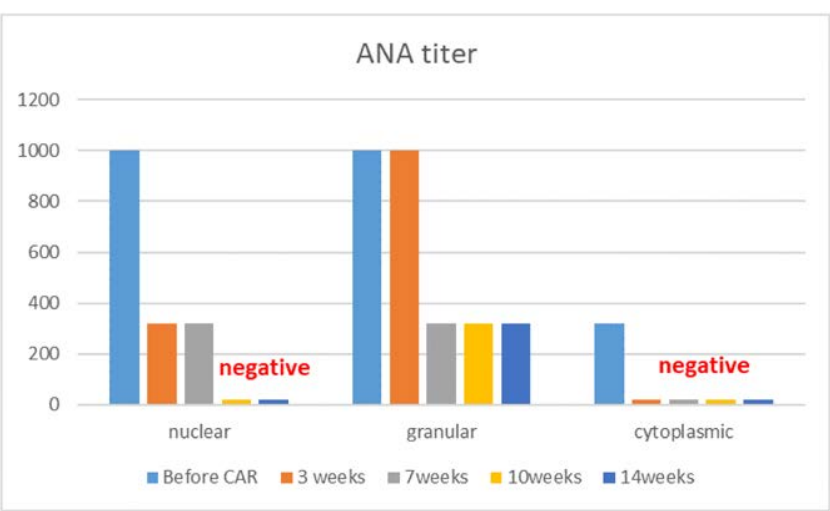

Figure 2. Reduction of different type of ANA titer at different time points.

Acknowledgments: patients and their families

Disclosure of Interests: Fang liu: None declared, Hongyu Zhang: None declared, Xiao Wang: None declared, Jia Feng: None declared, Yuanzhen cao Employee of: Employee of iCell Gene Therapeutics LLC, Yi Su: None declared, Masayuki Wada Employee of: employee of iCell Gene Therapeutics LLC, Yu Ma Employee of: employee of iCAR Bio Therapeutics Ltd, Yupo Ma Shareholder of: shareholder of iCell Gene Therapeutics LLC DOI: 10.1136/annrheumdis-2020-eular.4065

\begin{tabular}{|l|l}
\hline THU0037 & SURVIVIN INHIBITS TRANSCRIPTION OF PBX1 AND \\
& SUPPORTS THE EFFECTOR PHENOTYPE OF THE \\
MEMORY CD4 T CELLS IN RHEUMATOID ARTHRITIS
\end{tabular}

K. M. Andersson ${ }^{1}$, M. Erlandsson ${ }^{2}$, N. Oparina ${ }^{2}$, A. Damdimopoulos ${ }^{3}$, M. Jensen ${ }^{4}$, M. J. Garcia-Bonete ${ }^{4}$, G. Katona ${ }^{4}$, M. I. Bokarewa ${ }^{2} .{ }^{1}$ Göteborg university, Rheumatology and inflammation research, Göteborg, Sweden; ${ }^{2}$ the Sahlgrenska Academy at University of Gothenburg, Rheumatology and 